Revue de droit comparé du travail et de la sécurité sociale

$2 \mid 2020$

La vie personnelle du salarié

\title{
La protection de la vie privée des travailleurs, un défi pour la législation mexicaine
}

Gabriela Mendizábal Bermúdez

\section{OpenEdition}

1 Journals

Édition électronique

URL : https://journals.openedition.org/rdctss/1071

DOI : $10.4000 /$ rdctss. 1071

ISSN : 2262-9815

Éditeur

Centre de droit comparé du travail et de la sécurité sociale

Édition imprimée

Date de publication : 1 juin 2020

Pagination : 48-55

ISSN : 2117-4350

Référence électronique

Gabriela Mendizábal Bermúdez, «La protection de la vie privée des travailleurs, un défı pour la

législation mexicaine », Revue de droit comparé du travail et de la sécurité sociale [En ligne], 2 | 2020, mis en ligne le 01 novembre 2021, consulté le 11 novembre 2021. URL : http://journals.openedition.org/ rdctss/1071; DOI : https://doi.org/10.4000/rdctss.1071

\section{cc)}

Revue de droit comparé du travail et de la sécurité sociale est mise à disposition selon les termes de la Licence Creative Commons Attribution - Pas d'Utilisation Commerciale - Pas de Modification 4.0 International. 


\section{LA PROTECTION DE LA VIE PRIVÉE DES TRAVAILLEURS, UN DÉFI POUR LA LÉGISLATION MEXICAINE}

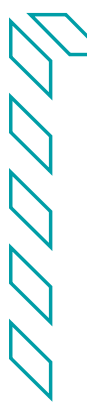

\section{Gabriella MendizÁbal Bermúdez}

Professeur-chercheur, Faculté de droit et de sciences sociales de l'Université Autonome de l'État du Morelos, Mexique

\section{RÉSUMÉ}

Ce chapitre traite des droits généraux des travailleurs et analyse les normes juridiques destinées à protéger la vie personnelle des salariés. Pour ce faire, il étudie les critères d'embauche, la conciliation entre vie professionnelle et vie familiale, et l'impact du travail sur le temps libre du salarié. Enfin, des conclusions sont formulées sur la base des sources d'informations utilisées.

Mots-clés : Travailleur, conciliation de la vie familiale et professionnelle, vie privée

\section{ABSTRACT}

This chapter deals with the general rights that workers have in labor matters, as well as analyzing the legal norms that protect the personal life of the worker, seeing it in three aspects: criteria for their employment; reconciliation of work-family life; and the impact of work on the worker's free time. Finally, the respective conclusions and research sources are established.

Key words: Worker, Reconciliation of Family and Work Life, Private Life 
e Mexique figure comme l'un des pays pionniers en matière de droit du travail et de protection sociale des salariés. Dès 1917, l'article 123 de la Constitution prévoyait une protection spécifique des droits des travailleurs : durée de la journée de travail, repos dominical, prime d'ancienneté, vacances, etc. Cependant, les limites de cette législation sont apparues au fil du temps face aux nouveaux défis auxquels les travailleurs ont été confrontés, notamment s'agissant de la place du travail dans la vie personnelle des salariés, et de l'impact de la vie professionnelle sur la vie privée - largement accentué, bien sûr, par l'usage des technologies.

Le cadre normatif mexicain en matière de réglementation du travail personnel et subordonné s'articule, pour l'essentiel, autour de deux normes juridiques : la Constitution politique des États-Unis du Mexique, à travers son célèbre article 123 qui garantit la jouissance des principaux droits et obligations des travailleurs dans les secteurs privé et public, et la Loi fédérale du travail, qui aborde en détails toutes les questions objectives et subjectives liées au travail.

Parmi elles, figurent notamment:

- la durée maximale de la journée de travail fixée à huit heures ;

- le salaire minimum ;

- l'égalité des salaires ;

- le droit à la participation aux bénéfices ;

- les heures supplémentaires ;

- le droit à la création de syndicats, le droit de grève ;

- le droit à la sécurité sociale ;

- le droit à un jour de repos tous les six jours ouvrables ${ }^{1}$.

Cependant, il existe actuellement un vide juridique qui pose la question de l'influence de la sphère professionnelle sur la vie personnelle du salarié dont les activités personnelles seraient incompatibles avec le travail. Cet écueil est principalement dû à l'évolution du contexte social, ainsi qu'aux technologies en tant que telles, et en particulier à la numérisation. Il est ainsi nécessaire d'analyser le cadre juridique mexicain en ce qui concerne la protection de la vie personnelle du travailleur dans l'exercice de ses fonctions professionnelles, afin d'évaluer la compatibilité de ces deux aspects essentiels de la vie de la personne.

Cette analyse repose sur trois axes fondamentaux qui visent à démontrer l'influence et l'existence possible d'une incompatibilité juridique entre vie professionnelle et vie personnelle, faute de législation appropriée. Néanmoins, il convient de mentionner que la plus haute instance du pouvoir judiciaire fédéral au Mexique, la Cour suprême de justice de la Nation, s'est prononcée dans certains cas, par le biais de sa jurisprudence et de plusieurs décisions, en faveur de la protection d'aspects importants de la vie personnelle du travailleur.

1 Constitution politique des États-Unis du Mexique, article 123. 
Cet article propose une analyse des normes juridiques qui protègent la vie personnelle des salariés, en particulier les critères d'embauche (I), la conciliation entre vie professionnelle et vie familiale (II), et l'impact du travail sur le temps libre du travailleur (III).

\section{I - CRITÈRES DE SÉLECTION DES TRAVAILLEURS}

L'article premier de la Constitution mexicaine interdit toute discrimination fondée, entre autres, sur le sexe, l'âge, le handicap et la religion. De même, la loi fédérale du travail dispose que le travail décent est un travail effectué sans aucune forme de discrimination, conformément aux dispositions de la Constitution elle-même. Ainsi, pour pouvoir être embauché, conserver son emploi ou bénéficier d'une promotion, les employeurs doivent respecter les normes susmentionnées. Nonobstant ce qui précède, il existe certaines exceptions au titre desquelles la vie personnelle du travailleur souhaitant être embauché ou conserver son emploi - peut influencer la décision de l'employeur à son égard. Parmi ces exceptions, on peut citer les tatouages (A), la grossesse (B) ou encore le VIH (C).

\section{A - LeS TATOUAGES OFFENSANTS, INCOMPATIBLES AVEC LE TRAVAIL}

Les travailleurs portant des tatouages ne peuvent faire l'objet d'une discrimination au travail. La Cour considère en effet, qu'en principe, le port de tatouages est un droit correspondant à l'exercice du libre développement de la personnalité et de la liberté d'expression. Les travailleurs tatoués souhaitant être embauchés - ou conserver leur emploi - ne sauraient donc faire l'objet d'une discrimination. Il en va de même concernant les procédures de licenciement ${ }^{2}$. Ainsi, le cadre légal garantit la protection du libre développement de la personnalité d'un travailleur tatoué.

Cependant, des exceptions existent. La Cour suprême de justice de la Nation, par le biais du recours d'amparo direct 465/2018, a considéré que le port et l'exhibition de tatouages dans l'enceinte de l'entreprise devraient être autorisés par la loi, et ne pas constituer un motif de discrimination au travail. En revanche, si le tatouage est une croix gammée, synonyme de haine raciste (antisémite), et affiché dans l'enceinte de l'entreprise, devant des salariés ou des dirigeants de confession juive, la protection constitutionnelle ne saurait être invoquée, car il est porté atteinte à la dignité, à l'égalité, à la sécurité et à la liberté d'expression des victimes, qui n'ont aucune obligation légale de tolérer cette situation ${ }^{3}$. Par conséquent, le fait de porter durant les heures de travail un tatouage incitant à la haine, sans le dissimuler de manière à ne pas porter atteinte aux droits d'autrui tels que la dignité humaine, la liberté et la sécurité, constitue un motif de licenciement valable et non discriminatoire.

\section{B - DISCRIMINATION AU TRAVAIL EN RAISON DE LA GROSSESSE}

Au Mexique, la discrimination dans l'entreprise en raison de la grossesse est récurrente à l'égard des femmes qui souhaitent entrer dans la vie active, conserver leur emploi ou bénéficier d'une promotion. Si l'article 1 de la Constitution mexicaine interdit toute

2 Thèse 1a. CXX/2019 (10a.), Séminaire Judiciaire de la Fédération et sa Gazette, Dixième époque, Livre 73, décembre 2019, p. 331.

3 Amparo Directo en Revisión 4865/2018, Ponente Norma Lucía Piña Hernández, Session 30 d'octobre 2019. 
discrimination fondée sur le sexe, il existe encore des cas de licenciement sans motif valable de femmes se trouvant dans cette période de vulnérabilité.

La Cour suprême de justice de la Nation a formulé des critères jurisprudentiels afin de protéger les travailleuses enceintes. L'un d'entre eux est issu d'une décision de janvier 2020, qui énonce que lorsqu'une travailleuse de confiance, enceinte, est licenciée sans motif valable au vu de la réglementation en vigueur, il doit être présumé que le licenciement avait pour motif son état de grossesse, sauf preuve contraire ${ }^{4}$. Dans le même esprit, la Cour suprême du Mexique estime qu'une travailleuse enceinte doit bénéficier d'un jugement qui intègre la perspective du genre, si pour son licenciement l'employeur invoque trois absences non justifiées sur une période de trente jours. Les juges doivent alors se prononcer en intégrant la perspective du genre. Autrement dit, la cause des absences doit faire l'objet d'une analyse approfondie, étant entendu que les absences dues à une grossesse n'ont pas à être justifiées par un certificat médical. Seuls l'état de santé et les circonstances de la grossesse sont pris en compte dans le cadre d'une étude spécifique "à caractère raisonnable ", afin d'analyser la situation en veillant au respect des droits fondamentaux de la mère et du fœtus ${ }^{5}$. Ainsi, ces critères jurisprudentiels ont pour objectif de protéger les femmes dans le domaine du travail et d'améliorer l'équilibre entre leur vie professionnelle et familiale, puisque pour des raisons naturelles, les femmes ont tendance à être plus vulnérables au travail que leurs homologues masculins.

\section{C - DiscriminATION LIÉE AU VIH}

Selon la jurisprudence de la Cour suprême de justice de la Nation, l'article 226, alinéa 45, de la loi de l'Institut de Sécurité Sociale des Forces Armées Mexicaines (ISSFAM) qui autorise la réforme des militaires pour inaptitude en raison de leur séropositivité, constitue une violation de l'article premier de la Constitution fédérale mexicaine.

En effet, d'après la jurisprudence établie, le législateur, en cherchant à protéger le rôle des Forces Armées, de ses membres et des tiers, a implicitement été discriminatoire vis-à-vis des individus licenciés en raison de cette maladie. Ce cadre légal est donc tout à fait inconstitutionnel, car discriminatoire dans la mesure où le fait d'être atteint du VIH est une raison suffisante pour permettre le licenciement des militaires ${ }^{6}$.

L'article 226 de la loi ISSFAM a fait l'objet de plusieurs réformes. Cependant, et malgré le fait que la Cour suprême de justice de la Nation se soit prononcée sur la question, les dispositions de l'alinéa visé n'ont jamais été modifiées.

\section{II - CONCILIATION VIE PROFESSIONNELLE ET VIE FAMILIALE}

La conciliation entre la vie professionnelle et la vie familiale constitue l'une des questions les plus mal encadrées par la législation mexicaine, car il n'existe aucune réglementation

4 Thèse I.16o.T.52 L (10a.), Séminaire Judiciaire de la Fédération et sa Gazette, Dixième époque, janvier 2020.

5 Thèse : XVII.1 1.C.T.74 L (10a.), Séminaire Judiciaire de la Fédération et sa Gazette, Livre 70, septembre 2019, p.1909.

6 Thèse : P./J.131/2007 (9a.), Séminaire Judiciaire de la Fédération et sa Gazette, Tome XXVI, décembre 2007, septembre 2019, p.12. 
sur le sujet. L'absence d'une législation veillant à une répartition des tâches domestiques et intégrant la perspective du genre, constitue de ce fait une violation des droits des femmes comme des hommes.

La Constitution énonce pourtant, que «les femmes et les hommes sont égaux devant la loi. La loi protège l'organisation et le développement de la famille ». Autrement dit, il existe un principe que la législation doit respecter, celui de l'intérêt supérieur de la famille. Cependant, l'absence d'harmonie législative entre les droits et obligations familiales et professionnelles, affaiblit la suprématie de ce principe. Il est donc nécessaire d'analyser certaines décisions visant à permettre la conciliation entre vie professionnelle et vie familiale par exemple eu égard au des parents d'avoir un service de garde pour les enfants (A) ou d'avoir droit à un congé parental (B).

\section{A - DROIT DES PARENTS DE BÉNÉFICIER DE SERVICES DE GARDERIE POUR ENFANTS}

La fourniture de service de garde d'enfants est née du besoin des femmes de s'intégrer sur le marché du travail, que ce soit pour évoluer professionnellement, par nécessité ou pour les deux. Toutefois, ce besoin s'exprime désormais de la part de tous les parents qui travaillent, les pères étant concernés au même titre que les mères. Il existe dans le domaine professionnel une ségrégation des hommes, qui ne bénéficient ni du même traitement, ni des mêmes avantages que les mères actives; leurs enfants font également l'objet de davantage de discriminations par rapport à ceux des mères qui travaillent (comme vu ci-après).

L'article 201 de la loi sur la sécurité sociale (LSS) dispose : "Afin de pallier le risque de ne pas pouvoir s'occuper des enfants en bas âge pendant la journée de travail, les services de garderie accordent les prestations énoncées au présent chapitre aux enfants des femmes actives et à ceux des travailleurs veufs ou divorcés, ou à qui la garde des enfants a été légalement confiée ${ }^{7}$. Cette loi est donc discriminatoire, puisqu'elle accorde le droit de bénéficier des services de garderie aux enfants des femmes qui travaillent sans autre condition que celle d'être mères actives alors que, pour en bénéficier, les hommes doivent être veufs ou disposer de la garde légale de l'enfant.

Cela signifie que, même s'ils sont affiliés à l'Institut mexicain de sécurité sociale (IMSS), les pères sont contraints d'inscrire leurs enfants dans des établissements privés, ce qui constitue une violation du droit de l'enfant de bénéficier des services de garderie contribuant à son développement et à son épanouissement, tel qu'énoncé dans la Convention relative aux droits de l'enfant ${ }^{8}$.

À ce titre, il convient de noter que la Cour suprême de justice de la Nation s'est prononcée sur la question des garderies de l'IMSS : "En imposant des exigences différentes aux femmes et aux hommes affiliés à ce service, le droit à l'égalité est violé »" Cette résolution dénonce clairement le fait que la législation ne permet pas aux hommes qui travaillent de bénéficier des services de garderies, et constitue une violation du principe

7 Loi de la sécurité sociale, article 201.

8 Convention relative aux droits de l'enfant, art. 18, § 3 .

9 Thèse 2a. CXXXIII/2016(10ª), Séminaire Judiciaire de la Fédération et sa Gazette, Dixième Époque, Deuxième Salle, Livre 37, décembre 2016, Tome I, p. 909. 
de non-discrimination, du droit à l'égalité entre les hommes et les femmes, du droit à la sécurité sociale et de l'intérêt supérieur de l'enfant ».

\section{B - CONGÉ PARENTAL}

Au cours du deuxième trimestre 2019, un décret a été publié au Journal officiel de la Fédération afin de permettre aux pères et aux mères qui travaillent de bénéficier $d^{\prime} u n$ congé pour s'occuper de leurs enfants de moins de seize ans atteints d'un cancer, quel qu'il soit, notamment lorsque ceux-ci ont besoin de repos ou sont hospitalisés durant les périodes critiques du traitement ${ }^{10}$.

Ainsi, les parents concernés bénéficient d'un congé pour pouvoir prendre soin de leur enfant en cas d'hospitalisation durant le traitement médical ; la durée maximale du congé est de 28 jours et celui-ci peut être accordé autant de fois que nécessaire, sans dépasser 364 jours sur une période maximale de trois ans ${ }^{11}$. II garantit au parent qui travaille de pouvoir s'occuper de son enfant mineur atteint de cette maladie, sans que cela ait des conséquences sur son emploi. Malheureusement, pour l'instant, ces congés concernent uniquement les enfants atteints de cancer. II faut espérer qu'à l'avenir, d'autres types de maladies nécessitant tout autant la présence des parents aux côtés de leur enfant, comme la sclérose en plaques, seront prises en compte.

\section{III - TEMPS LIBRE - ENTREPRISE ET INTIMITÉ AU TRAVAIL}

La Constitution politique des États-Unis du Mexique n'énonce pas explicitement le droit à l'intimité de la vie privée, mais elle reconnaît certains droits qui lui sont associés et qui le garantissent partiellement. Parmi eux, l'article 16 protège trois aspects de la vie privée : il prévoit que nul ne saurait être dérangé par toute autorité compétente sans un avis écrit préalable, et protège l'inviolabilité des communications comme celle des correspondances. Il s'agit de garantir le principe de légalité qui pose une limite aux autorités en les obligeant à respecter la vie privée des personnes.

Cependant, les outils technologiques qui nécessitent l'usage d'Internet, comme la vidéosurveillance (A), le courrier électronique (B), les applications de messagerie de type WhatsApp ou les appels vidéo, ne sont pas pris en compte, ni leur impact sur l'environnement de travail.

\section{A - LA VIDÉOSURVEILLANCE AU TRAVAIL}

La surveillance des travailleurs au Mexique est une pratique récurrente des entreprises ou des employeurs. En 2013, l'ancien Institut fédéral d'accès à l'information publique (aujourd'hui « Institut national pour la transparence, l'accès à l'information et la protection des données personnelles ») a publié un document énonçant diverses recommandations visant à ce que les entreprises ayant recours à la vidéosurveillance - ou à tout autre système

10 Journal Officiel de la Fédération, Décret ajoutant diverses dispositions à la loi sur la sécurité sociale, à la loi de l'Institut de la sécurité sociale et des services aux travailleurs de l'État, et à la loi fédérale du travail, Mexique, 2019 : https://dof.gob.mx/nota detalle. php?codigo $=5561817 \&$ fecha $=04 / 06 / 2019$

11 Loi de la sécurité sociale, art. 140. 
du même genre - garantissent la protection des données personnelles de leurs salariés, en précisant l'existence de ces mécanismes et en publiant un bref avis de confidentialité pour la vidéosurveillance ${ }^{12}$.

II s'agit de protéger les travailleurs, car ce type de pratiques constitue une violation et une atteinte à leur vie privée dès lors qu'ils permettent de connaître le comportement et la conduite des salariés sur leur lieu de travail. En ce sens, il est évident que ces recommandations protègent l'intégrité des salariés et leur droit à la vie privée. Néanmoins, en l'absence de cadre légal, cela ne suffit pas. La question de la protection du droit à la vie privée des travailleurs sur le lieu de travail reste donc ouverte.

\section{B - ATteINTE À LA VIE PRIVÉE DES TRAVAILLEURS PAR LE BIAIS DU COURRIER ÉLECTRONIQUE}

L'article 135, section IX de la loi fédérale du travail interdit aux salariés d'utiliser les outils et les équipements fournis par l'employeur à des fins autres que celles auxquelles ils sont destinés.

Les employeurs fournissent à leurs salariés, à des fins opérationnelles, des outils tels que des ordinateurs et des comptes de messagerie électronique pour leur permettre de mener à bien leurs activités au sein de l'entreprise. Cependant, les courriers électroniques ne se limitent pas aux activités professionnelles et sont couramment utilisés dans le cadre de correspondances privées par le travailleur lui-même.

Dans ce contexte, il convient de noter que le droit à la vie privée constitue l'un des droits de l'homme reconnus dans la Constitution politique mexicaine ${ }^{13}$ et dans la Déclaration universelle des droits de l'homme $^{14}$, qui le considèrent toutes deux comme un droit inviolable. De même, la Cour suprême de justice de la Nation a énoncé que personne n'était légitime à intercepter le courrier électronique d'un tiers, y compris en utilisant pour ce faire un ordinateur qui ne lui appartient pas ${ }^{15}$. Par conséquent, le fait que l'employeur ait accès aux comptes de messagerie électronique de ses salariés (en particulier les comptes professionnels) lui donne le droit de les consulter. La réglementation mexicaine sur cette question est floue et source de confusion; il est donc nécessaire de mettre en place une législation simple et appropriée à ce type de situation, afin de garantir la protection du droit à la vie privée du travailleur.

En conclusion, le défi que représente pour la législation mexicaine la mise en place de politiques et d'avantages visant la protection intégrale du travailleur en tant qu'être humain, se heurte à un problème de nature économique et se traduit donc par l'absence d'accords visant à créer de nouvelles réglementations ou à réformer plus globalement les réglementations existantes.

12 Voir «La surveillance des employés, une atteinte à la vie privée ? „, Forbes México, Mexique, 2013 : https://www.forbes.com.mx/el-monitoreo-de-empleados-una-invasion-a-la-privacidad/

13 Constitution politique des États-Unis du Mexique, article 16.

14 Déclaration universelle des droits de l'homme, article 12.

15 Thèse 1a. CLX/2011(9a), Séminaire Judiciaire de la Fédération et sa Gazette, Neuvième époque, Première salle, Livre 37, Août 2011, Tome XXXIV, p. 217. 
Face à cette réalité, la Cour a contribué à établir différents critères pour protéger la vie privée des salariés au travail. Toutefois, cette protection ne vaut que pour les cas d'espèce soumis; c'est pourquoi, les réformes juridiques sont indispensables.

L'un des plus grands défis pour le Mexique sera d'intégrer dans sa législation les deux principes suivants :

- Le principe d'une stabilité renforcée de l'emploi : ce droit garantit de pouvoir accéder à un emploi - ou de le conserver - aux personnes fragilisées d'une manière ou d'une autre en raison de difficultés personnelles, par exemple la maladie d'une personne économiquement dépendante ou à charge, ou la naissance d'un enfant. Si cet événement ne devrait pas être considéré comme une situation de vulnérabilité en soi, il expose malheureusement au risque de licenciement. Le principe d'une stabilité renforcée de l'emploi consiste à garantir que tout travailleur puisse conserver son travail et bénéficier des avantages salariaux et sociaux correspondants, même contre la volonté de l'employeur, en l'absence de motif pertinent justifiant le licenciement ${ }^{16}$.

- Le principe de l'intérêt supérieur de la famille : considérée comme une institution, la famille joue un rôle important à différents niveaux de la société; par exemple, dans les domaines économique, social, culturel, juridique, politique et civil. Pour préserver son équilibre, il est donc nécessaire de la protéger. En tant que pièce maîtresse de l'organisation de l'État, c'est aussi la famille qui répond aux besoins fondamentaux de ses membres en préparant leur participation à la vie sociale. Une législation permettant de concilier de façon adéquate la vie professionnelle et la vie familiale est urgente si l'on veut atteindre cet objectif.

C'est pourquoi, il est essentiel de protéger la vie personnelle et intime du salarié au travail, via une protection garantie par le cadre juridique national, et non par des critères jurisprudentiels ou des décisions telles que celles analysées ci-dessus.

16 «Stabilité renforcée de l'emploi », Gerencie, Mexique, 2019 : https://www.gerencie.com/estabilidadlaboral-reforzada.html 